\title{
Instagram como herramienta de aprendizaje en el aula universitaria
}

\author{
Daniel Belanche ${ }^{\mathrm{a}}$, Narciso Lozano ${ }^{\mathrm{b}}$ y Alfredo Pérez-Rueda ${ }^{\mathrm{c}^{*}}$ \\ a Departamento de Dirección de Marketing e Investigación de Mercados, Facultad de Economía y \\ Empresa, Universidad de Zaragoza, Gran Vía 2, Zaragoza 50005, España; mail belan@unizar.es \\ b Departamento de Dirección de Marketing e Investigación de Mercados, Facultad de Economía y \\ Empresa, Universidad de Zaragoza, Gran Vía 2, Zaragoza 50005, España; mail nlozano@unizar.es \\ c Departamento de Dirección de Marketing e Investigación de Mercados, Facultad de Ciencias \\ Sociales y Humanas, Universidad de Zaragoza, Ciudad Escolar S/N, Teruel, 44003 España; mail \\ aperu@unizar.es
}

\begin{abstract}
The growing interest and use of Instagram as a marketing tool is a great opportunity to include it in learning processes related to this area. This research is based on the creation of an Instagram account for a subject of a Marketing and Market Research University Degree. The study, conducted over two years, describes the evolution of students from a passive role, as mere passive users of the account of the subject, to a more active role, in which they managed the account and generated content. In this way, the project contributes to analyze the evolution of an activity that tries to renew the traditional pedagogical processes and expand the temporal and spatial focus of the student towards a more practical orientation. Results suggest that students perceive a greater complexity in the activity when they administer the Instagram account. However, students also perceive higher levels of satisfaction and activity recommendation when they are in charge of managing the account and creating the content. In addition, managing the account in the social network also contributes to improving learning outcomes in transversal competences such as the use of digital tools and the promotion of creativity.
\end{abstract}

Keywords: Instagram, Product, Brand, Marketing, Social media Participation.

\section{Resumen}

El creciente interés y uso de Instagram como herramienta de marketing supone una oportunidad para los procesos de aprendizaje afines a éste área. Esta investigación se basa en la creación de una cuenta de Instagram para una asignatura del Grado universitario de Marketing e Investigación de Mercados. El estudio, realizado durante dos años, describe la evolución de 
los estudiantes desde un rol pasivo, siendo meros usuarios pasivos de la cuenta de la asignatura, a un rol más activo, en el que administraban la cuenta y generaban contenidos. De esta manera, el proyecto contribuye a analizar la evolución de una actividad que trata de renovar los procesos pedagógicos tradicionales y expandir el enfoque temporal y espacial del estudiante hacia una orientación más práctica. Los resultados sugieren que los estudiantes perciben una mayor complejidad en la actividad cuando administran la cuenta de Instagram. Sin embargo, los estudiantes también perciben niveles más altos de satisfacción y recomendación de la actividad cuando son los encargados de gestionar la cuenta y crear el contenido. Además, administrar la cuenta en la red social también contribuye a mejorar los resultados de aprendizaje en competencias transversales tales como el uso de herramientas digitales y el fomento de la creatividad.

Palabras clave: Instagram. Producto. Marca. Marketing. Redes sociales. Participación.

\section{Introducción}

Desde hace varios años el mundo empresarial busca lograr una vinculación mayor y más directa con sus posibles clientes. En este sentido, el uso de las redes sociales como herramienta de comunicación y gestión de marca genera cada vez más interés tanto en ámbito profesional como en el académico (Zhang y otros, 2017). Así, desde el punto de vista empresarial, y más concretamente desde el del marketing, la literatura previa establece que las redes sociales permiten generar un valor añadido en el consumidor, atrayéndolo y obteniendo beneficios derivados de su participación (Mangold y Faulds, 2009).

Entre las numerosas redes sociales que podemos encontrar, Instagram está experimentando un gran crecimiento en relación al número marcas que utilizan esta red social para promocionar sus productos (Forbes, 2018a). De la misma forma, esta red social ha crecido rápidamente en cuanto a su número de usuarios, especialmente entre los jóvenes (Casaló Flavián, e Ibáñez-Sánchez, 2017; IAB 2107) alcanzando recientemente la cifra de 1 billón de usuarios activos (Forbes, 2018b). Su carácter hedónico y visual son determinantes para justificar la expansión que está experimentando en los últimos años (Belanche, Cenjor y Pérez-Rueda 2019; Casaló Flavián, e Ibáñez-Sánchez, 2017).

Instagram es una red social que permite a sus usuarios la creación de imágenes y vídeos que son compartidos para que otros usuarios puedan ver e interactuar con ese contenido. Los usuarios suelen seguir las publicaciones de sus marcas de referencia, líderes de opinión (influencers o más específicamente instagrammers) y amigos que emiten valoraciones acerca de determinados productos y marcas a través de esta red social. Así pues, el alumno

(cc) BY-NC-ND 2019, Universitat Politècnica de València 
de marketing puede acceder en tiempo real a información de calidad sobre una empresa, producto o servicio (Casaló Flavián, e Ibáñez-Sánchez, 2018). Además, se ha señalado que Instagram genera un mayor engagement en comparación a otras redes sociales como Facebook o Twitter (Locowise, 2017).

Además, el éxito del crecimiento de Instagram viene dada por un aumento de los usuarios de Smartphone y una notable mejora de la calidad de sus cámaras. Los Smartphones son dispositivos ampliamente utilizados por la sociedad actual y prueba de ello es que dos terceras partes de la población mundial cuenta con uno (Wearesocial, 2018). Estos teléfonos permiten a los usuarios acceder vía online a un mayor volumen de información de forma instantánea (Hubert y otros, 2017), a la vez que incrementan el engagement en el proceso de aprendizaje (Domingo y Garganté, 2016). Estas características hacen que estos dispositivos sean considerados adecuados y muy útiles a la hora de llevar a cabo actividades en el aula.

Nuestra investigación trata de solventar la necesidad de utilizar herramientas que permitan vincular los contenidos de las asignaturas con aspectos actuales y más prácticos que el alumnado puede encontrar en su entorno habitual de forma sencilla y atractiva. Por otro lado, la utilización de una red social en el aula como herramienta de aprendizaje, como es el caso de Instagram, permite a los alumnos adquirir competencias de comunicación y análisis que serán muy útiles en su futuro profesional.

\section{Objetivos}

Teniendo en cuenta las consideraciones previas, esta investigación que ha sido llevada a cabo durante dos cursos académicos, trata de contribuir mediante la incorporación de actividades vinculadas a Instagram, a la renovación de los procesos pedagógicos de asignaturas con un eminente enfoque instrumental. Nuestra investigación trata de aprovechar la relación entre el impacto empresarial de la red y su relación con los usuarios más jóvenes con el objetivo de mejorar el aprendizaje y la motivación de los estudiantes con la asignatura Decisiones sobre Producto y Marca, que aborda temáticas muy vinculadas con la gestión de la imagen de marca (por ej. logotipos, posicionamiento, lanzamiento de nuevos productos, etc.) en Instagram.

En segundo lugar, este proyecto apuesta por mejorar la comprensión de los contenidos teóricos e incrementar el interés y vinculación de los estudiantes con la asignatura mediante herramientas atractivas y de uso fuera del aula. Instagram permite que los alumnos relacionen contenidos explicados en el aula con aspectos actuales y prácticos que pueden encontrar en su entorno habitual de forma más sencilla y atractiva.

En tercer lugar, se pretende mejorar el manejo de herramientas tecnológicas y el aprendizaje colaborativo de los estudiantes mediante la creación y el análisis crítico de contenidos generados por marcas y líderes de opinión. Instagram facilita al alumno el acceso en tiempo real a información de calidad (p. ej., siguiendo los perfiles de marcas y líderes de opinión en un sector determinado). En este sentido, el uso de Instagram como herramienta de aprendizaje permite que los estudiantes adquieran competencias de 
comunicación y análisis muy útiles en su futuro profesional. Así pues, se desarrolla una experiencia innovadora apoyada en el uso de nuevas tecnologías (Instagram, Smartphones, uso de medios audiovisuales y etiquetas para redes sociales, etc.) mediante la cual los estudiantes generan contenidos vinculados a la asignatura y analizan críticamente el contenido generado por otros agentes relevantes en el proceso de gestión y comunicación de marca.

En cuarto y último lugar, la utilización de Instagram en la asignatura representa un canal de interacción adicional con el profesorado, ayudando a valorar de forma conjunta elementos de la actualidad vinculados a las decisiones sobre productos y marcas. Instagram representa un canal de interacción con otras personas interesadas en la misma temática (p. ej. alumnos de otros cursos del Grado en Marketing e Investigación de Mercados o de otros grados englobados dentro de la misma área temática como el Grado en Administración y Dirección de Empresas).

\section{Desarrollo de la innovación}

Como se ha explicado anteriormente, Instagram supone una importante herramienta de comunicación en la actualidad, favoreciendo la diferenciación y gestión de productos y marcas para las empresas. Además, esta red social tiene una clara vinculación con la sociedad más joven (Casaló Flavián, e Ibáñez-Sánchez, 2017). Por ello, su utilización para la consolidación de conocimientos relacionados con la asignatura, así como de competencias profesionales básicas resulta claramente innovadora y oportuna.

La investigación se ha llevado a cabo durante dos cursos académicos 2017-2018 y 20182019. La asignatura en la que se enmarca la investigación es Decisiones sobre Producto y Marca. Esta asignatura es de carácter obligatorio en el tercer curso del Grado en Marketing e Investigación de Mercados de la Universidad de Zaragoza y forma parte del módulo de Variables de Marketing.

Durante el tercer curso académico los estudiantes del grado comienzan a familiarizarse con las herramientas de marketing, tanto desde la perspectiva de las acciones del marketing mix (producto, precio, distribución y comunicación), como de instrumentos concretos (p. ej. aplicaciones informáticas) que permitan gestionar dichas herramientas.

Durante el primer curso, se creó una cuenta de Instagram para la asignatura (@productounizar, Figura 1). La cuenta se utilizó para seguir los perfiles de 87 marcas con el propósito de observar y analizar el uso que realizan las empresas de esta herramienta para la gestión de la imagen de producto y marca (p. ej. nuevos lanzamientos de productos, posicionamiento de marca, generación de notoriedad, comparación con la competencia, etc.). Puntualmente, también se siguieron a instagrammers muy vinculados a temas relacionados con producto y marca (p. ej. nutricionistas que comentan etiquetas de los productos). El segundo año el número de perfiles seguidos aumento a 144 marcas.

Durante el primer año del proyecto la cuenta @productounizar estuvo siempre gestionada por los profesores de la materia y llegó a tener 48 seguidores, en su mayoría estudiantes de 
la asignatura, aunque entre sus seguidores también se podían encontrar otros alumnos del grado o alguna de las marcas seguidas. En el segundo curso la responsabilidad de gestionar la cuenta paso a ser de los alumnos de la asignatura y se alcanzaron los 117 seguidores, encontrando entre ellos estudiantes del Grado en Marketing de diferentes cursos, personal y cuentas institucionales de la Universidad de Zaragoza y otros usuarios externos interesados en la materia.

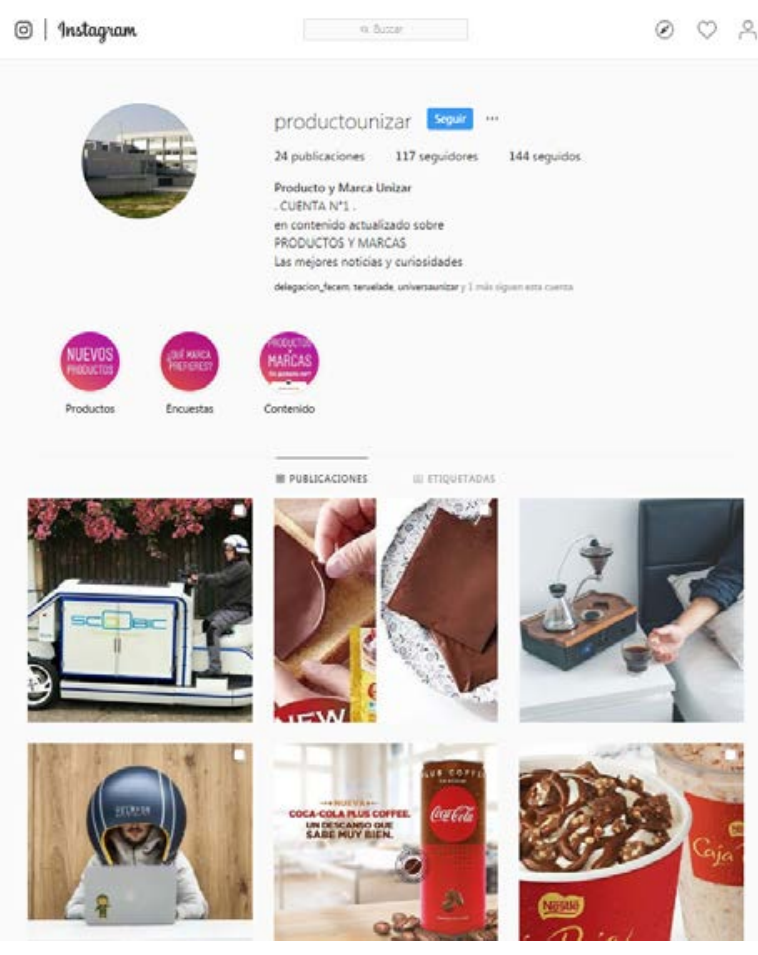

Fig. 1 Cuenta @productounizar en Instagram 2018-2019

Durante ambos cursos, se realizó una primera sesión informativa, en la que se explicaron las actividades que los alumnos iban a llevar a cabo en el proyecto y el contexto del que surge esta herramienta de marketing. Además, se ponía especial interés en que todos los alumnos supieran cómo crear y usar una cuenta, así como en definir las etiquetas que iban a ser utilizadas durante las actividades.

En el curso 2017-2018, los estudiantes publicaron en Instagram aquella información (p. ej. la nueva imagen de una marca a la que seguían) relacionada con los temas de la asignatura que consideraron relevantes. Los alumnos debían etiquetar en cada publicación la cuenta de la asignatura (@productounizar), o bien escribir un “hashtag” creado exclusivamente para la misma (\#productounizar). Además, en los comentarios de la publicación debían analizarse brevemente qué concepto del temario estaba relacionado (p. ej. una promoción de Spotify dirigida específicamente a estudiantes durante el periodo de exámenes; Figura 2). Esta tarea se debía realizar fuera del horario de clase. Posteriormente, el resto de alumnos y el profesorado, desde la cuenta oficial de la asignatura, podían difundir o valorar brevemente esa información en la propia red social. Se logró que aproximadamente la 
mitad de los estudiantes siguieran la cuenta, sin embargo, se trataba de una participación pasiva, ya que apenas creaban contenidos o utilizaban la etiqueta. A modo de ejemplo y para estimular la creación de contenido, el profesorado subió contenidos que lograron bastante visibilidad e interés, pero que no consiguieron una participación activa por parte del alumnado en general.

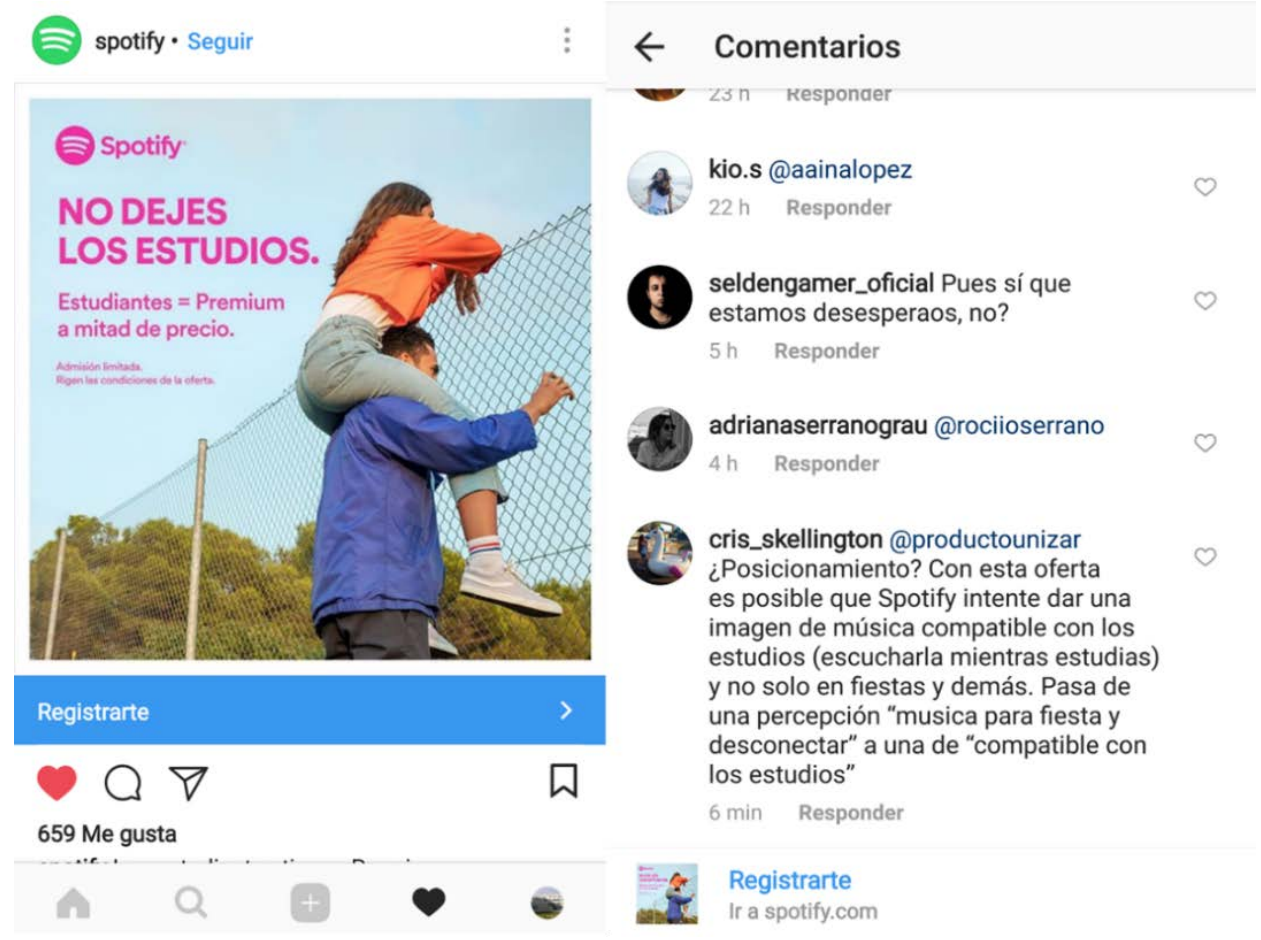

Fig. 2 Participación de alumnos en la actividad 2017-2018

Por otro lado, cada dos semanas, al comienzo de la sesión dedicada a las actividades prácticas de la asignatura, se comentaron en clase las publicaciones o cuestiones más relevantes publicadas en términos de su relevancia en relación a los contenidos de la asignatura. De esta forma, y siguiendo la metodología que se emplea en las clases prácticas, el profesorado llevaba a clase los contenidos que se habían generado durante ese periodo. Del mismo modo, durante estas sesiones se analizaba la información publicada por cuentas de marcas (p. ej. el Corte Inglés, Nocilla, etc.), el tipo de formato utilizado (p. ej. muro, Instagram Stories, etc.) y la vinculación con en temario de la asignatura (p. ej. creación de un nuevo producto, posicionamiento hacia un segmento de población, etc.).

Al finalizar el primer curso, se realizó una encuesta de evaluación específica, a través de SurveyMonkey, sobre la actividad a los 53 estudiantes asistentes en el último día de clase.

En cambio y como principal diferencia, durante el curso 2018-2019, los alumnos eran los encargados de administrar la cuenta y crear el contenido desde la cuenta @productounizar. De esta manera, el papel del alumno evolucionaba de un uso pasivo y marginal a uno más proactivo y con mayor responsabilidad. Los estudiantes gestionaron la cuenta en grupos formados por dos o tres personas, administrando cada grupo la cuenta durante una semana 
(ampliada a dos semanas en caso de festivos). El alumno seleccionaba, adaptaba y creaba contenidos que consideraba interesantes y que estaba vinculado con la asignatura (Figura 3). Para ello, hubo que establecer previamente unas normas mínimas que les impedían, por ejemplo, modificar contenido de terceros o grabar en espacios comerciales sin permiso.

Al igual que el curso anterior, cada dos semanas, se dedicaba una sesión práctica a comentar y debatir sobre las publicaciones o cuestiones más relevantes del contenido que habían creado los alumnos.

Finalmente, al finalizar el cuatrimestre del curso 2018-2019 se realizó la misma encuesta de evaluación específica sobre la actividad a través de SurveyMonkey. En esta ocasión 46 alumnos acudieron a la última sesión de la asignatura y respondieron a la encuesta.

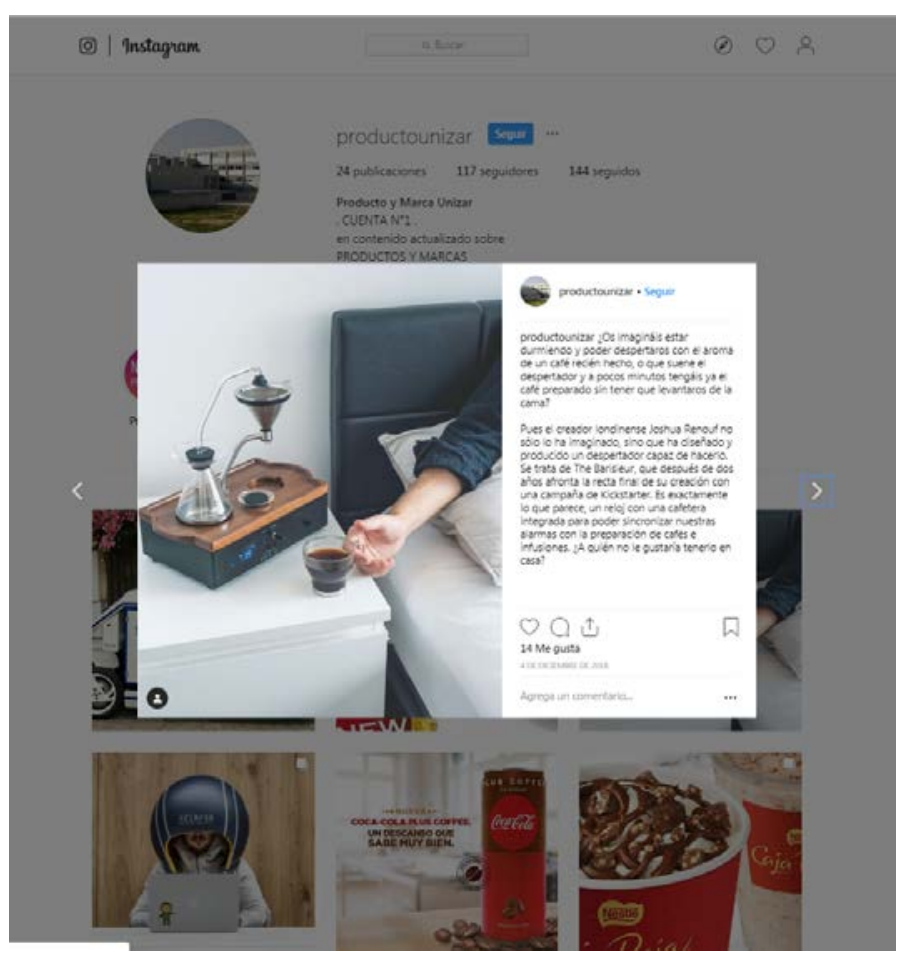

Fig. 3 Creación de contenido llevada a cabo por los alumnos en la actividad 2018-2019

En ambos cursos, el hecho de disponer de todo un cuatrimestre también permitió seguir la evolución experimentada por las cuentas profesionales seguidas y observar las respuestas que se daban entre ellas en términos competitivos (p. ej. empresas que comercializan el mismo producto). Al finalizar el curso, se realizó una sesión final a modo resumen de toda la actividad desarrollada en la cuenta, prestando especial interés en aquellas acciones que habían evolucionado a lo largo del tiempo. 


\section{Resultados}

La encuesta de satisfacción y consecución de objetivos del proyecto de innovación docente fue realizada por 53 estudiantes el primer año y 46 el segundo año, obteniendo un total de 99 cuestionarios válidos. De todas las respuestas válidas obtenidas el 32,32 \% fueron hombres y el 67,68 restante mujeres. La edad más común entre los participantes en la investigación fue de 21 a 25 años. Las respuestas a las preguntas de la encuesta de evaluación final sobre la actividad sugieren importantes conclusiones y lecciones para los próximos cursos. Las escalas de medida utilizadas para la realización del cuestionario fueron obtenidas de diferentes fuentes basadas en el contenido teórico y los objetivos de la investigación. Concretamente, cada una de las variables de nuestra investigación fue medida con escalas basadas en Belanche, Casaló y Flavián (2011), así como en Orús y otros, (2016).

La Figura 4 muestra la comparación de los principales resultados de la actividad en ambos años.

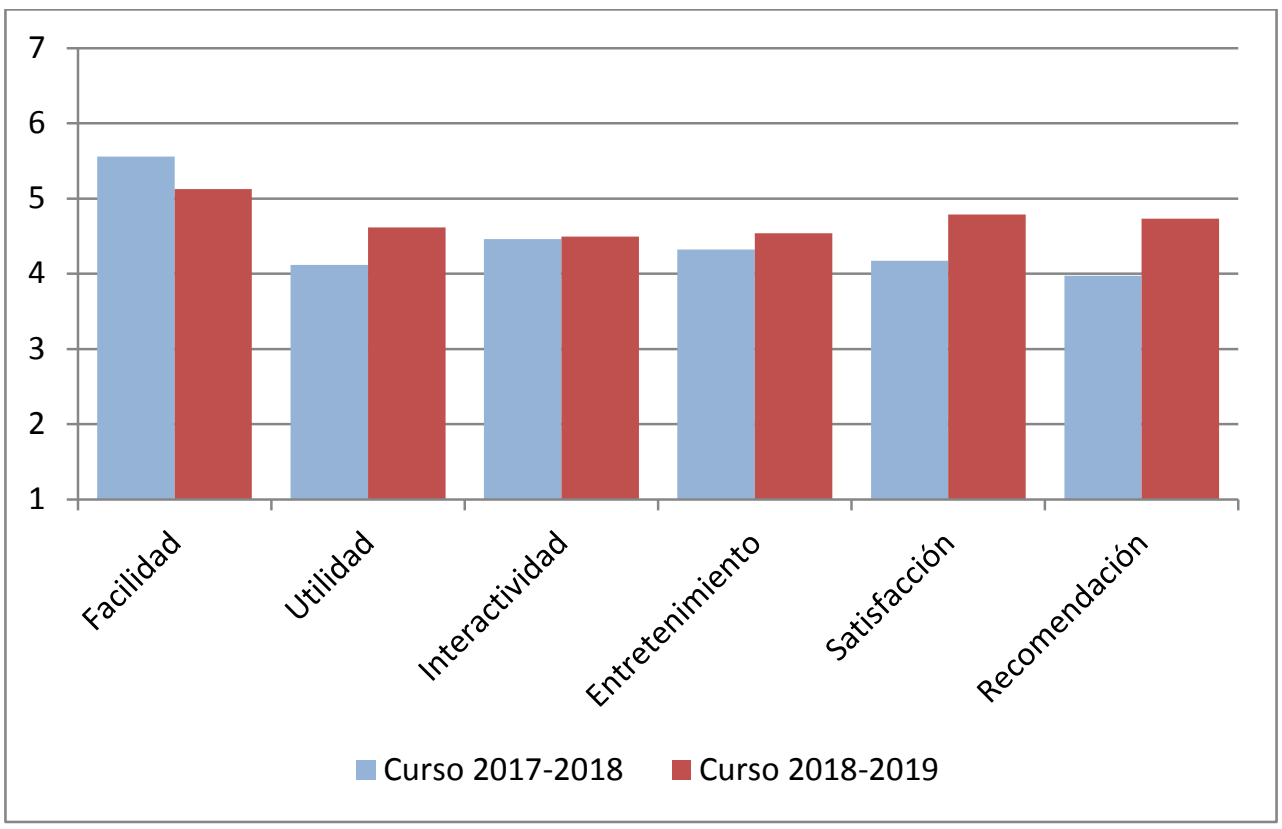

Fig. 4 Principales resultados de la actividad

Los estudiantes han considerado que la actividad era más difícil el segundo año, aunque no de manera significativa $\left(\mathrm{M}_{\text {FacilidadCurso2017-2018 }}=5.56, \mathrm{M}_{\text {FacilidadCurso2018-2019 }}=5.12 ; t(97)=\right.$ $1.51, p>0.10$ ). Este resultado es comprensible, ya que la gestión integral de la cuenta durante el segundo año de investigación ha conllevado una serie de dificultades que no pudieron darse el primer año, cuando la cuenta era gestionada por el profesorado. Por otro lado, los alumnos encuentran la actividad más útil $\left(\mathrm{M}_{\text {UtilidadCurso2017-2018 }}=4.12\right.$, $\left.\mathrm{M}_{\text {UtilidadCurso2018-2019 }}=4.62 ; t(97)=1.53, p>0.10\right)$, más interactiva $\left(\mathrm{M}_{\text {InteractividadCurso2017-2018 }}=\right.$ 4.46, $\left.\mathrm{M}_{\text {InteractividadCurso2018-2019 }}=4.49 ; t(97)=0.11, p>0.10\right) \mathrm{y}$ más entretenida 
$\left(\mathrm{M}_{\text {EntretenimientoCurso2017-2018 }}=4.32, \mathrm{M}_{\text {EntretenimientoCurso2018-2019 }}=4.54 ; t(97)=0.68, p>0.10\right)$ cuando han tenido que gestionar la cuenta, aunque las diferencias no son estadísticamente significativas. La actividad sí que ha mejorado significativamente el segundo año en cuanto a la satisfacción percibida al realizarla $\left(\mathrm{M}_{\text {SatisfacciónCurso2017-2018 }}=4.17, \mathrm{M}_{\text {SatisfacciónCurso2018-2019 }}=\right.$ 4.79; $t(97)=1.90, p<0.10$ ). Del mismo modo, este segundo año ha registrado una mejora significativa en cuanto a la intención de recomendar la actividad $\left(\mathrm{M}_{\text {RecomendaciónCurso2017-2018 }}=\right.$ 3.97, $\left.\mathrm{M}_{\text {RecomendaciónCurso2018-2019 }}=4.73 ; t(97)=2.45, p<0.05\right)$.

Por último, la Figura 5 muestra, los resultados ofrecen una mejora significativa en cuanto a la capacidad de aprendizaje de los alumnos en lo que se conoce habitualmente como adquisición de competencias transversales.

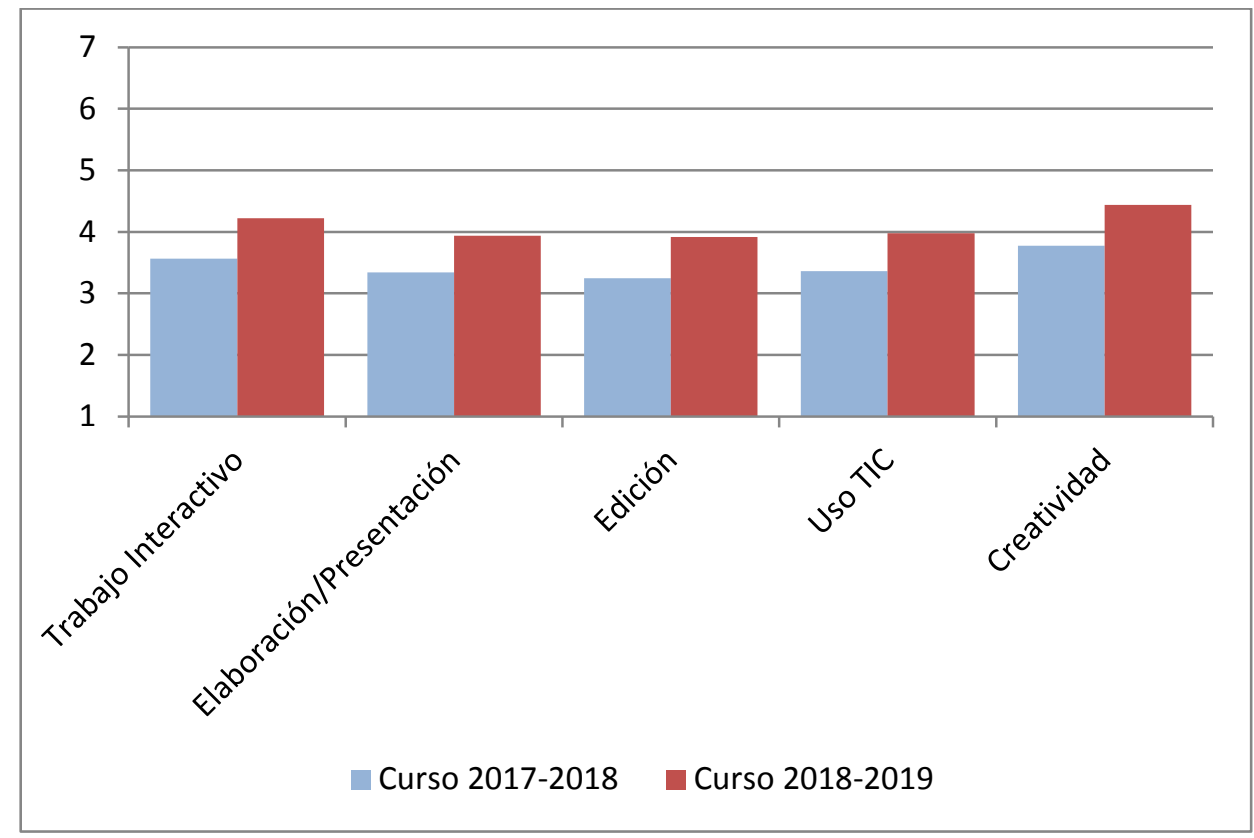

Fig. 5 Principales resultados de aprendizaje (competencias) derivados de la actividad

Especialmente, los alumnos que gestionaron la cuenta de la asignatura creen que la actividad contribuyó a mejorar su capacidad de trabajar de manera interactiva $\left(\mathrm{M}_{\text {TrabajoInteractivoCurso2017-2018 }}=3.57, \mathrm{M}_{\text {TrabajoInteractivoCurso2018-2019 }}=4.21 ; t(97)=2.09, p<0.05\right)$; la elaboración y presentación de ideas en público ( $\mathrm{M}_{\text {ElaboraciónPresentaciónCurso2017-2018 }}=3.34$, $\left.\mathrm{M}_{\text {ElaboraciónPresentaciónCurso2018-2019 }}=3.93 ; t(97)=1.77, p<0.10\right)$; la capacidad para usar herramientas de edición de imagen $\left(\mathrm{M}_{\text {EdiciónCurso2017-2018 }}=3.25\right.$, $\mathrm{M}_{\text {EdiciónCurso2018-2019 }}=3.91$; $t(97)=1.92, p<0.10)$; la capacidad para usar las Tecnologías de la Información y Comunicación (TIC) $\left(\mathrm{M}_{\text {TICCurso2017-2018 }}=3.36, \mathrm{M}_{\text {TICCurso2018-2019 }}=3.98 ; t(97)=1.83, p<\right.$ 0.10); así como la capacidad creativa $\left(\mathrm{M}_{\text {CreatividadCurso2017-2018 }}=3.77, \mathrm{M}_{\text {CreatividadCurso2018-2019 }}=\right.$ $4.43 ; t(97)=1.88, p<0.10)$. 


\section{Conclusiones}

En general, este proyecto de innovación docente ha logrado el objetivo de contribuir a la renovación de los procesos pedagógicos, tal y como muestran los resultados del estudio realizado en estos dos años. Por otro lado, se ha conseguido el objetivo de mejorar el manejo de la herramienta y contribuir al aprendizaje colaborativo, sobre todo el uso profesional en la gestión de producto y marca.

La actividad desarrollada para esta investigación ha permitido que se complementaran los procesos pedagógicos habituales mediante la inclusión de una actividad vinculada a una red social de uso cotidiano por alumnos y empresas (particularmente en gestión de productos y marcas) como es Instagram. se ha facilitado el aprendizaje de los aspectos teóricos de la asignatura, dándole al alumno un papel más activo tanto en la elaboración de contenido como en su valoración. Además, se ha ayudado a que el alumno mejoré competencias transversales (innovación, comunicación, creatividad, trabajo en equipo) vitales en el ámbito profesional. Por último, se ha fomentado el aprendizaje colaborativo y la interacción entre estudiantes, profesorado y un entorno práctico real.

En general, se ha logrado implantar el sistema propuesto de manera fácil y correcta. El seguimiento de perfiles empresariales de marcas resulta útil para obtener ejemplos reales de gestión que puedan ser aprendidos durante las clases prácticas o fuera del aula. La consecución de los objetivos marcados ha sido posible gracias a:

1. La planificación previa y la estimulación continuada por parte del profesorado.

2. El conocimiento previo que tienen los estudiantes de la red social (la inmensa mayoría ya eran usuarios de Instagram).

3. La constante creación de contenidos audiovisuales en la red social por parte de las marcas.

Sin embargo, durante el primer curso en el que se llevó a cabo esta investigación, la actividad generada en términos de nuevos contenidos subidos a la red por los estudiantes fue insuficiente. Se logró que aproximadamente la mitad de los estudiantes siguieran la cuenta, pero se trataba de una participación más bien de carácter pasivo. Los alumnos apenas creaban contenidos, utilizaban el nombre de la cuenta o el "hashtag” creados para este proyecto. A modo de ejemplo y para estimular la creación de contenido, el profesorado subió contenidos que lograron bastante visibilidad e interés, pero que no consiguieron una participación activa y masiva por parte del alumnado. Esta falta de participación pudo deberse a una mayor dificultad percibida por el alumnado para generar, compartir y comentar contenidos que la que se esperaba en esta actividad.

Por ello, el segundo año en el que se llevó acabo ese proyecto, se propuso que los alumnos gestionaran la cuenta y tuvieran la responsabilidad de crear los contenidos. En base a la experiencia previa del profesorado en otros proyectos de innovación docente, se creyó que los estudiantes tendrían un comportamiento similar al registrado en otras redes sociales como Twitter. Sin embargo, aunque la participación respecto al año pasado fue mayor, la creación de contenidos en Instagram es ligeramente más compleja, más visual y menos formal que en otras redes sociales, por lo que los estudiantes pudieron ver mayores 
dificultades para participar activamente (p. ej., búsqueda de información, síntesis y publicación, redacción comercial, etc.).

De manera general, los resultados señalan que los estudiantes perciben una mayor complejidad en la actividad cuando tienen la responsabilidad de administran la cuenta de Instagram y crear los contenidos que se van a publicar. A su vez, es interesante comprobar que, de la misma manera, cuando los estudiantes son responsables de la cuenta perciben mayores niveles de satisfacción y recomendación. Por otro lado, los resultados muestran que administrar la cuenta en la red social también mejora los resultados de aprendizaje en competencias transversales tales como el uso de herramientas digitales y el fomento de la creatividad, respecto al año anterior.

Además, la participación en esta actividad estuvo desvinculada de la calificación de la asignatura. La actividad quería estar enmarcada dentro del interés voluntario de los estudiantes y de esta manera intentar que se vinculara más a su propia actividad en redes que a la evaluación de la asignatura. A su vez, la incorporación de la participación como actividad de evaluación hubiera supuesto establecer criterios objetivos de evaluación difíciles de calificar (p. ej. cantidad, calidad, contenido, etc.) e incorporarlos a la guía docente (para lo cual es necesario mayor previsión y estabilidad a medio plazo).

Así pues, la falta de evaluación de la actividad probablemente resultó un elemento desmotivador para los estudiantes que suelen guiarse por criterios utilitarios (Tejedor, 2003). Se trata por tanto de una acción a modo de prueba, que seguramente debería mejorarse y afianzarse antes de considerar su inclusión como actividad de evaluación; si bien, sería recomendable incluirla para que los estudiantes aprecien más claramente el valor y la vinculación de la actividad con la asignatura.

\section{Transversalidad}

Este estudio realizado durante los dos últimos años pretende seguir realizándose durante los próximos cursos. Para ello, y tras el análisis de los resultados obtenidos, se han considerado que la participación debería incluirse de alguna forma dentro de las actividades de evaluación (quizá, para evitar cambios en la guía docente, se podría considerar la posibilidad de ser tenida en cuenta como una actividad práctica más). Por otro lado, con el objetivo de lograr una mayor sostenibilidad se han tenido en cuenta otros aspectos a implementar.

En primer lugar, al tratarse de una cuenta abierta, se podría fomentar que otros estudiantes del Grado de Marketing e Investigación de Mercados (o de otros englobados dentro de la misma área temática como el Grado de Administración y Dirección de Empresas, etc.) puedan seguirla, o que los estudiantes de la asignatura continúen siguiendo la cuenta una vez que hayan superado la misma.

En segundo lugar, también se ha planteado la posibilidad de gestionar la cuenta de forma conjunta con otras asignaturas, en particular con la asignatura de Comunicación Comercial. 
En definitiva, Instagram es una herramienta que aúna intereses de gestión de marca y producto, pero también de comunicación online.

Estos intereses son comunes a otras asignaturas similares en otros grados (p. ej. Grado de Administración y Dirección de Empresas), por lo que la propuesta podría ser implantada en asignaturas afines, así como en otras universidades o en aquellas materias que utilicen las redes sociales como apoyo al proceso de enseñanza-aprendizaje. De esta forma, sería posible la creación de una comunidad interdisciplinar formada por alumnos de diferentes grados con intereses comunes que pudieran poner en contacto diferentes puntos de vista con respecto a la temática abordada en la cuenta de Instagram. Además, teniendo en cuenta que Instagram es una de las redes sociales más utilizadas por los jóvenes en la actualidad, es de esperar que los alumnos tengan una participación en la cuenta al estar interesados en los contenidos que se publiquen.

Por lo tanto, el presente proyecto de innovación docente sería de gran interés para asignaturas que aborden temáticas vinculadas a la imagen de marca o la gestión de la comunicación en un contexto social. Este proyecto podría ser empleado en aquellas áreas con un mayor interés por la elaboración y difusión de contenidos audiovisuales vinculados a la imagen y el marketing, así como en la difusión de contenidos por agentes relevantes en el proceso comunicativo.

\section{Referencias}

Belanche, D., Casaló, L. V., y Flavián, C. (2011). “Online Public Services Adoption: An Analysis Integrating TAM and TPB Models" en European Journal of Management and Business Economics, vol. 20 (4), p. 41-55.

Belanche, D., Cenjor, I., y Pérez-Rueda, A. (2019, en prensa). "Instagram Stories versus Facebook Wall: an advertising effectiveness analysis" en Spanish Journal of Marketing-Esic.

Casaló, L.V., Flavián, C. e Ibáñez-Sánchez, S. (2017). “Antecedents of consumer intention to follow and recommend an Instagram account" en Online Information Review, vol 41, p. 10461063.

Casaló, L.V., Flavián, C. e Ibáñez-Sánchez, S. (2018, en prensa). "Influencers on Instagram: Antecedents and consequences of opinion leaderhip" en Journal of Business Research.

Domingo, M. G. y Garganté, A. B. (2016). “Exploring the use of educational technology in primary education: Teachers' perception of mobile technology learning impacts and applications' use in the classroom” en Computers in Human Behavior, vol. 56, p. 21-28.

Forbes (2018a). “As Facebook shifts, Instagram emerges as a new home for brands”. 1 de Febrero. bit.ly/2OPIpwY.

Forbes (2018b). “How Instagram is eating the world” 25 de junio. bit.ly/2qbgEjB.

Hubert, M., Blut, M., Brock, C., Backhaus, C. y Eberhardt, T. (2017). “Acceptance of smartphone based mobile shopping: Mobile benefits, customer characteristics, perceived risks, and the impact of application context” en Psychology \& Marketing, vol. 34, p. 175-194.

IAB (2017). Estudio anual de redes sociales 2017. IAB Spain. Madrid.

Locowise (2017). “Instagram follower growth is now lagging behind Facebook page likes growth” 18 de abril. bit.ly/2MJ7wvG. 
Mangold, W. G. y Faulds, D.J. (2009). “Social media: The new hybrid element of the promotion mix” en Business Horizons, vol. 52, p. 357-365.

Orús, C., Barlés, M. J., Belanche, D., Casaló, L., Fraj, E., y Gurrea, R. (2016). “The effects of learner-generated videos for YouTube on learning outcomes and satisfaction" en Computers \& Education, vol. 95, p. 254-269.

Tejedor, F.J.T. (2003). "Poder explicativo de algunos determinantes del rendimiento en los estudios universitarios” en Revista española de pedagogía, vol. 61, p. 5-32.

Wearesocial (2018). “Digital in 2018: World’s internet users pass the 4 billion mark” 30 de enero. bit.ly/2HkIRQe.

Zhang, M., Guo, L., Hu, M. y Liu, W. (2017). "Influence of customer engagement with company social networks on stickiness: Mediating effect of customer value creation” en International Journal of Information Management, vol. 37, p. 229-240. 